Naeve-Stoß, Nicole; Kastrup, Julia; Herold, Gabriele

\title{
Praxisphasen im Rahmen des Studiums Lehramt an beruflichen Schulen - Gestaltung und Erfahrungen an der Universität Hamburg
}

Haushalt in Bildung \& Forschung 4 (2015) 1, S. 3-16

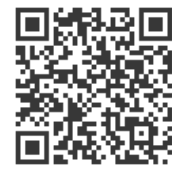

Quellenangabe/ Reference:

Naeve-Stoß, Nicole; Kastrup, Julia; Herold, Gabriele: Praxisphasen im Rahmen des Studiums Lehramt an beruflichen Schulen - Gestaltung und Erfahrungen an der Universität Hamburg - In: Haushalt in Bildung \& Forschung 4 (2015) 1, S. 3-16 - URN: urn:nbn:de:0111-pedocs-203591 - DOI:

10.25656/01:20359

https://nbn-resolving.org/urn:nbn:de:0111-pedocs-203591

https://doi.org/10.25656/01:20359

in Kooperation mit / in cooperation with:

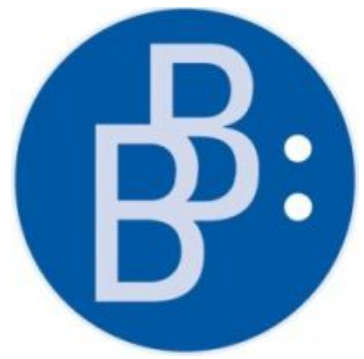

https://www.budrich.de

\section{Nutzungsbedingungen}

Gewährt wird ein nicht exklusives, nicht übertragbares, persönliches und beschränktes Recht auf Nutzung dieses Dokuments. Dieses Dokument ist ausschließlich für den persönlichen, nicht-kommerziellen Gebrauch bestimmt. Die Nutzung stellt keine Übertragung des Eigentumsrechts an diesem Dokument dar und gilt vorbehaltlich der folgenden Einschränkungen: Auf sämtlichen Kopien dieses Dokuments müssen alle Urheberrechtshinweise und sonstigen Hinweise auf gesetzlichen Schutz beibehalten werden. Sie dürfen dieses Dokument nicht in irgendeiner Weise abändern, noch dürfen Sie dieses Dokument für öffentliche oder kommerzielle Zwecke vervielfältigen, öffentlich ausstellen, aufführen, vertreiben oder anderweitig nutzen.

Mit der Verwendung dieses Dokuments erkennen Sie die Nutzungsbedingungen an.

\section{Terms of use}

We grant a non-exclusive, non-transferable, individual and limited right to using this document.

This document is solely intended for your personal, non-commercial use. Use of this document does not include any transfer of property rights and it is conditional to the following limitations: All of the copies of this documents must retain all copyright information and other information regarding legal protection. You are not allowed to alter this document in any way, to copy it for public or commercial purposes, to exhibit the document in public, to perform, distribute or otherwise use the document in public.

By using this particular document, you accept the above-stated conditions of use.

\section{Kontakt / Contact:}

\section{peDOCS}

DIPF | Leibniz-Institut für Bildungsforschung und Bildungsinformation Informationszentrum (IZ) Bildung

E-Mail: pedocs@dipf.de

Internet: www.pedocs.de

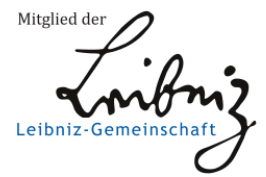




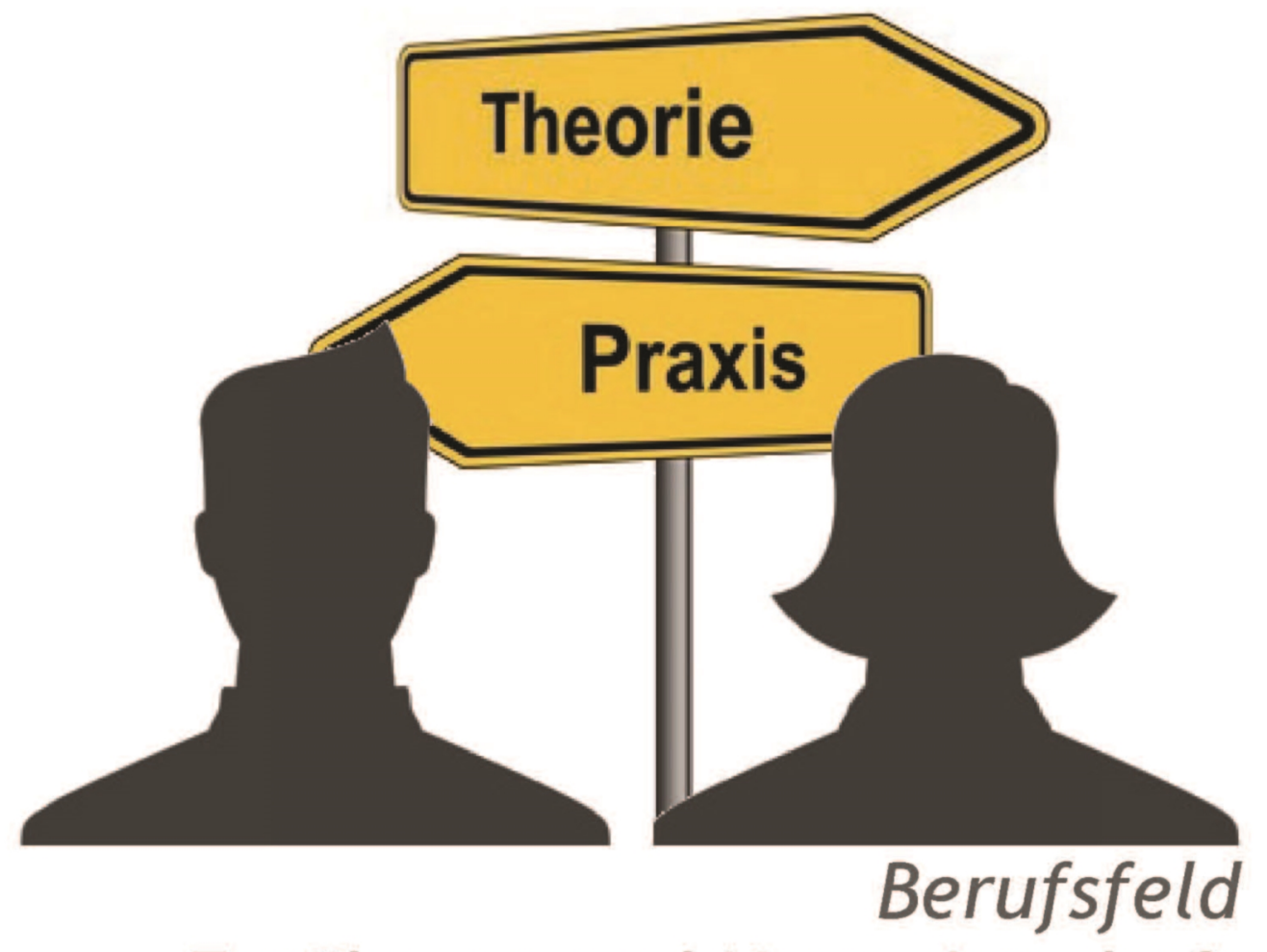

Ernährung und Hauswirtschaft

Transfer zwischen Theorie und Praxis 
Irmhild Kettschau

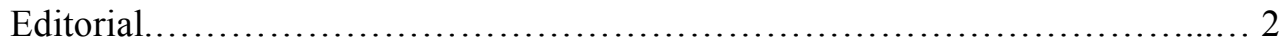

Nicole Naeve-Stoß, Julia Kastrup \& Gabriele Herold

Praxisphasen im Rahmen des Studiums Lehramt an beruflichen Schulen -

Gestaltung und Erfahrungen an der Universität Hamburg................................. 3

Sandra Mester

Das schulische Praxissemester in NRW: Konzept und Innovation am Institut

für Berufliche Lehrerbildung der Fachhochschule Münster....................... 17

Andrea Mohorič

Transfer von Modellversuchsergebnissen - ein Beispiel aus dem

Förderschwerpunkt Berufsbildung für nachhaltige Entwicklung

Karolin Wirth

Inklusion: Ansätze zur praktischen Umsetzung in der

beruflichen Lehrerbildung................................................. 40

Susanne Miesera \& Stefanie Sander

Sprache im Beruf in Bildungsgängen der beruflichen Fachrichtung

Ernährung und Hauswirtschaft.................................................... 54

Anna Hoff

Fachräume im berufsbildenden Unterricht - die Lehrküche als Beispiel

Helge Schulz

Bildungsgangarbeit in der Fachdidaktik

Alexandra Brutzer \& Christine Küster

Lernbereich Alltagskultur im Berufsfeld Ernährung und Hauswirtschaft -

Skizze für einen Orientierungsrahmen.

Julia Kastrup \& Irmhild Kettschau

Berufliche Lehrkräftebildung gestärkt -

Bundesarbeitsgemeinschaft gegründet. 108

Marie Nölle

Rezension: Berufsbildung für nachhaltige Entwicklung. 
Nicole Naeve-Stoß, Julia Kastrup \& Gabriele Herold

\section{Praxisphasen im Rahmen des Studiums Lehramt an beruflichen Schulen - Gestaltung und Erfahrungen an der Universität Hamburg}

An der Universität Hamburg haben die Studierenden des Lehramts an beruflichen Schulen in unterschiedlichen Lehrveranstaltungen über das Bachelor- und Masterstudium hinweg einen unmittelbaren Kontakt zum schulischen Praxisfeld. In diesem Beitrag werden die Praxisformate vorgestellt, wobei eine Konkretisierung der jeweiligen Studienangebote unter der spezifischen Perspektive der beruflichen Fachrichtung Ernährungs- und Haushaltswissenschaften erfolgt und insgesamt der Fokus der Betrachtung auf das Kernpraktikum, die Praxisphase im Masterstudium, gelegt wird.

Schlüsselwörter: Praxisphasen, Praxiserfahrungen, erfahrungsbezogene Reflexion, theoriegeleitete Reflexion

\section{Die berufliche Fachrichtung Ernährungs- und Haushaltswissenschaften an der Universität Hamburg}

Das Studium des Lehramts an beruflichen Schulen an der Universität Hamburg gliedert sich in einen sechssemestrigen Bachelor- und einen viersemestrigen Masterstudiengang. Beide Studiengänge setzen sich wiederum aus drei Teilstudiengängen zusammen:

- Teilstudiengang Erziehungswissenschaft einschließlich Fachdidaktik und Berufs- und Wirtschaftspädagogik,

- Teilstudiengang der beruflichen Fachrichtung,

- Teilstudiengang des Unterrichtsfaches.

Bezüglich der beruflichen Fachrichtungen kann zwischen den folgenden gewählt werden: Bau- und Holztechnik, Chemotechnik, Elektrotechnik/Informationstechnik, Ernährungs- und Haushaltswissenschaften, Gesundheitswissenschaften, Kosmetikwissenschaften, Medientechnik, Metalltechnik oder Wirtschaftswissenschaften. Im Rahmen der beruflichen Fachrichtung Ernährungs- und Haushaltswissenschaften wird das fachwissenschaftliche Studium der berufsfeldbezogenen naturwissenschaftlichen Grundlagen (z.B. Lebensmittelchemie, Ernährungswissenschaften, Mikrobiologie, Grundlagen der Arbeitswissenschaft, Betriebswirtschaft, Datenverarbeitung, Statistik, Ernährungs-, Hauswirtschafts- sowie Betriebswirtschaftslehre des Hotelund Gaststättengewerbes, Lebensmittelmikrobiologie und -hygiene sowie Lebens- 


\section{Praxisphasen für das Lehramt an beruflichen Schulen}

mittellehre) an der Hochschule für Angewandte Wissenschaften absolviert. Im Masterstudium ist eine Schwerpunktbildung, auch in Kombination mit Projektseminaren, möglich (Behörde für Schule und Berufsbildung/HIBB, 2014, S. 23).

Das fachdidaktische und das berufs- und wirtschaftspädagogische Lehrangebot werden vom Institut für Berufs- und Wirtschaftspädagogik der Fakultät für Erziehungswissenschaft der Universität Hamburg verantwortet. Die Zulassung zum Studium kann gemäß Prüfungsordnung in der Regel nur dann erfolgen, wenn eine abgeschlossene Berufsausbildung in der beruflichen Fachrichtung vorliegt oder ein zwölfmonatiges Betriebspraktikum absolviert wurde.

Seit der Einführung der neuen Studienstrukturen im WS 2007/2008 haben in der beruflichen Fachrichtung Ernährungs- und Haushaltswissenschaften zu jedem Wintersemester durchschnittlich 24 Studierende das Bachelor-Studium begonnen, im Masterstudium sind dies seit dem WS 2010/2011 durchschnittlich 17 Studierende. Aktuell (WS 2014/2015) sind in dieser Fachrichtung 130 Studierende eingeschrieben (davon 76 im Bachelor- und 54 im Masterstudiengang).

Die Studierenden im Studiengang Lehramt an beruflichen Schulen haben in unterschiedlichen Formaten während des gesamten Studiums einen unmittelbaren Kontakt zum schulischen Praxisfeld. In diesem Beitrag werden diese praxisbezogenen Studienangebote vorgestellt, wobei eine Konkretisierung der jeweiligen Angebote unter der spezifischen Perspektive der beruflichen Fachrichtung Ernährungs- und Haushaltswissenschaften erfolgt.

\section{Praxisformate im Bachelor- und Masterstudiengang}

Im Zuge der Umgestaltung der Studiengänge auf Bachelor- und Masterstrukturen wurden an der Universität Hamburg die schulischen Praxisphasen im Rahmen des berufs- und wirtschaftspädagogischen Studiums umgestaltet. Von Beginn des Bachelorstudiums an sind praxisbezogene Elemente integriert. Dabei wird ein Konzept verfolgt, das auf die Verknüpfung von wissenschaftlichem Wissen und erfahrungsbezogenem Handlungswissen abzielt. Demzufolge kommt sowohl dem eigenen Erleben im späteren Praxisfeld als auch der theoriegeleiteten Reflexion dieser Erfahrungen eine besondere Bedeutung zu (Naeve-Stoß, 2013; Riebenbauer \& NaeveStoß, 2013). Die Bedeutung dieser praxisbezogenen Studienangebote wird vor allem darin gesehen,

- dass die Studierenden den zentralen Gegenstand ihres Studiums - LehrLernprozesse im organisatorischen Rahmen beruflicher Schulen und/oder betrieblicher Ausbildung - aus unmittelbarer Anschauung erleben und aktiv erkunden sollen,

- dass sie aus der Konfrontation mit Praxis Probleme und Fragestellungen erkennen können, die sie in ihr Studium hineintragen und dort systematisch weiterverfolgen, 


\section{Praxisphasen für das Lehramt an beruflichen Schulen}

- dass sie die Tragfähigkeit, aber auch die Grenzen wissenschaftlicher Begriffe, Verfahren und Theorien in der Auseinandersetzung mit Praxis erkunden,

- dass sie durch eigenes Handeln in der Praxis ihre Fähigkeiten und ihr Wissen erproben, sich selbst in pädagogischen Situationen erleben und damit Anregungen für ihren weiteren Qualifizierungsprozess gewinnen,

- und schließlich, dass sie ihre Berufseignung überprüfen.

All dies soll nicht neben dem Studium, sondern muss in dieses integriert erfolgen. Der Vorbereitung, Begleitung und Reflexion der Praxiserfahrungen kommen dabei eine besondere Bedeutung zu.

Abbildung 1 gibt einen Überblick über die Praxisformate im Bachelor- und Masterstudiengang für das Lehramt an beruflichen Schulen an der Universität Hamburg: die praxisorientierte Einführung in die Berufs- und Wirtschaftspädagogik, das Orientierungspraktikum und das Kernpraktikum. Während die praxisorientierte Einführung in die Berufs- und Wirtschaftspädagogik sowie das Orientierungspraktikum fachrichtungsübergreifend stattfinden, ist das Kernpraktikum in großen Teilen fachrichtungsspezifisch organisiert. Die einzelnen Praxisformate werden im Folgenden dargestellt (Naeve-Stoß, 2013).

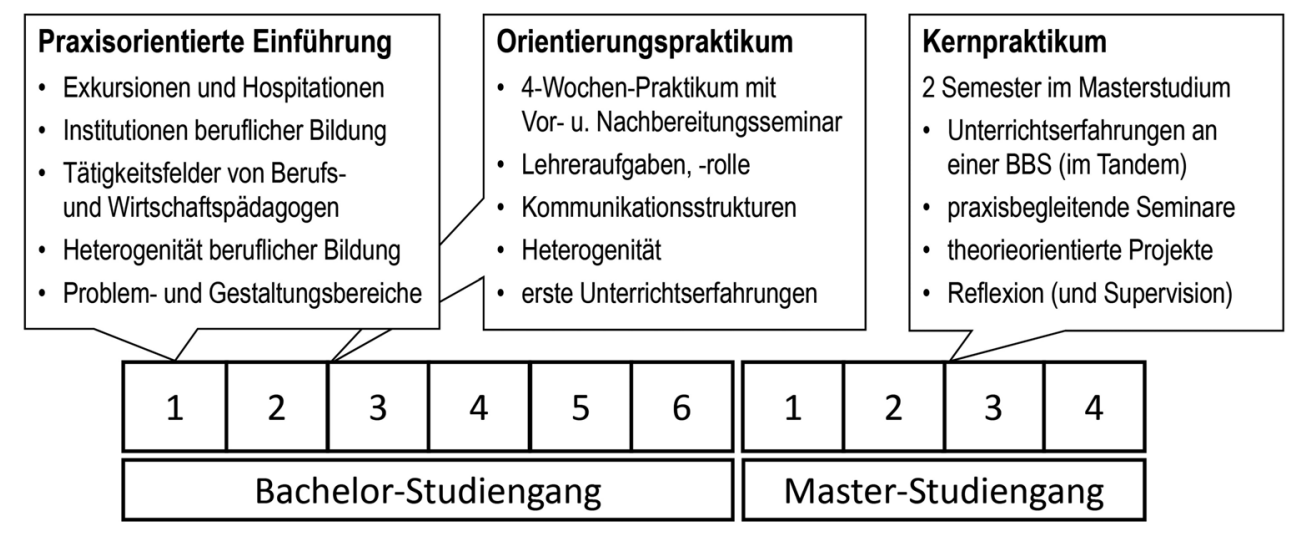

Abb. 1: Praxisphasen im Studium Lehramt an beruflichen Schulen in Hamburg

\subsection{Praxisorientierte Einführung in die Berufs- und Wirtschaftspädagogik (PE)}

Die Studierenden absolvieren diese Veranstaltung i. d. R. im ersten Semester ihres Bachelorstudiums als Teil des Moduls „Orientierung im berufs- und wirtschaftspädagogischen Studium“. Die Veranstaltung findet fachrichtungsübergreifend statt. Ein wichtiges Ziel besteht darin, den Studierenden sowohl die Anforderungen an eine professionelle berufs- bzw. wirtschaftspädagogische Tätigkeit in verschiedenen Praxisfeldern als auch die Heterogenität der Zielgruppen beruflicher Bildung und die 


\section{Praxisphasen für das Lehramt an beruflichen Schulen}

damit einhergehenden Anforderungen zu verdeutlichen. Vor diesem Hintergrund sollen sie eigene berufliche Interessen und Potenziale erkennen und reflektieren sowie hieraus Konsequenzen für den eigenen Professionalisierungsprozess und die individuelle Studiengestaltung ziehen. Damit die Studierenden eine Orientierung über verschiedene berufs- und wirtschaftspädagogische Arbeitsfelder und Bildungskonzepte erhalten, werden Hospitationen in unterschiedlichen Institutionen beruflicher Bildung durchgeführt, insbesondere an Berufsbildenden Schulen. In der Seminararbeit wird an bereits vorhandene Schul- und Berufserfahrungen der Studienanfänger angeknüpft. Durch systematische Vor- und Nachbereitung der jeweiligen Hospitationen wird bei den Studierenden ein doppelter Perspektivenwechsel angeregt: einerseits von der Schüler- zur Lehrersicht auf Unterricht und andererseits von der Wahrnehmung und Beurteilung auf der Grundlage voruniversitärer Alltagserfahrungen und -begriffe hin zur systematischen, theoriegeleiteten Wahrnehmung und Analyse vorfindlicher Praxis.

\subsection{Orientierungspraktikum (OP)}

In der vorlesungsfreien Zeit zwischen dem zweiten und dritten Semester des Bachelorstudiums findet ein vierwöchiges Orientierungspraktikum statt, das in das Modul „Erkundung im berufs- und wirtschaftspädagogischen Praxisfeld“ eingebettet ist, welches sich über das zweite und dritte Semester des Bachelor-Studiums erstreckt. Vorbereitet wird das Praktikum, das die Studierenden an einer Berufsbildenden Schule in Deutschland absolvieren, durch ein Seminar zur beruflichen Sozialisation, zu dessen inhaltlichen Schwerpunkten sowohl die Sozialisation von Jugendlichen in der beruflichen Bildung zählen als auch die spezifischen Sozialisationserfahrungen der Studierenden auf ihrem Weg zum Lehrerberuf gehören. Über das Orientierungspraktikum soll eine reflexive Auseinandersetzung mit der Lehrerarbeit und der eigenen Berufswahl angebahnt werden. Vorrangiges Ziel des Orientierungspraktikums ist es also, dass sich die Studierenden mit dem späteren beruflichen Handlungsfeld als Lehrkraft an einer Berufsbildenden Schule auseinandersetzen, sich dieses mithilfe theoretisch-begrifflicher Konzepte in seiner Vielfalt erschließen und vor diesem Hintergrund die eigene Berufswahl reflektieren.

\subsection{Kernpraktikum (KP)}

Im Rahmen des Masterstudiums nimmt das Kernpraktikum eine Schlüsselposition ein. Diese durch unterschiedliche universitäre Veranstaltungen begleitete Praxisphase umfasst insgesamt 30 Leistungspunkte (also das Volumen eines ganzen Semesters) und wurde vom Institut für Berufs- und Wirtschaftspädagogik und dem Landesinstitut für Lehrerbildung und Schulentwicklung (Abteilung Ausbildung Berufsbildende Schulen) gemeinsam entwickelt. Das Kernpraktikum erstreckt sich über das zweite und dritte Semester des Masterstudiums. Den zentralen Lernort für die Studierenden stellt eine Berufsbildende Schule in Hamburg oder eines benachbarten Bundeslandes dar, die im Berufsfeld der jeweiligen beruflichen Fachrichtung ausbildet. 


\section{Praxisphasen für das Lehramt an beruflichen Schulen}

Das Kernpraktikum gliedert sich in zwei Module, die sich wiederum auf fünf parallel verlaufende und sich über die gesamte Dauer des Praktikums erstreckende Bänder verteilen (vgl. Abbildung 2) (vertiefend z. B. Tramm \& Naeve, 2010; Tramm, Fahland \& Naeve, 2012):

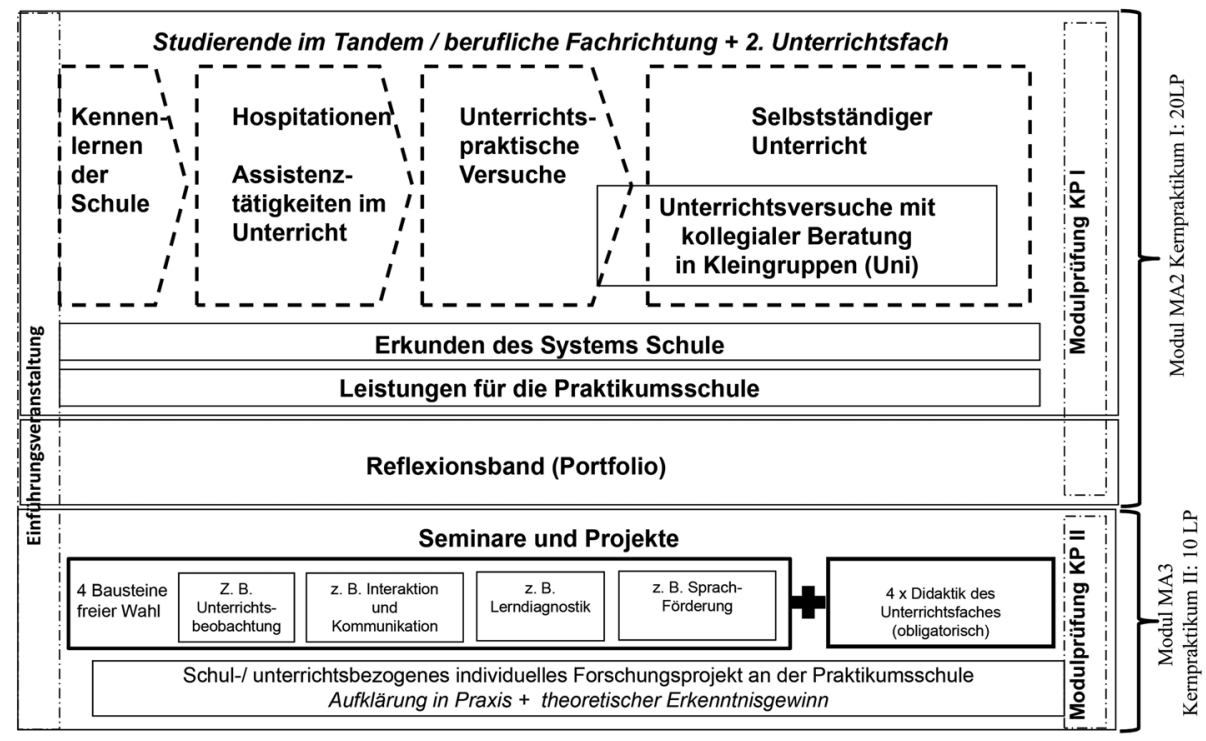

Abb. 2: Das Bändermodell des Kernpraktikums in Hamburg im Masterstudiengang Lehramt an beruflichen Schulen

Das Modul Kernpraktikum I umfasst das Schulband, ein begleitendes Reflexionsband sowie das Unterrichtsversuchsband. In dem Modul stehen die Auseinandersetzung mit Schule und Unterricht sowie die Entwicklung entsprechender analytischer, reflexiver und pragmatischer Kompetenzen im Zentrum. Das zweite Modul Kernpraktikum II umfasst das Seminarband und das Projektband.

\section{Schulband}

Das Schulband stellt das Zentrum des Kernpraktikums dar. Die Studierenden absolvieren jeweils zu zweit (im Tandem) das Kernpraktikum an einer Berufsbildenden Schule. Im zweiten Mastersemester sind sie in einem zeitlichen Umfang von einem Tag (Vormittag) während der Vorlesungszeit und während der vorlesungsfreien Zeit über einen Zeitraum von vier Wochen an vier bis fünf Tagen an der Schule; im dritten Mastersemester im Umfang von zwei bis drei Tagen (Vormittagen). Sie werden an den Schulen von Mentoren betreut, die im Rahmen einer Fortbildungssequenz durch das Landesinstitut für Lehrerbildung und Schulentwicklung (Abteilung Ausbildung Berufsbildende Schulen) in Zusammenarbeit mit dem Institut für Berufsund Wirtschaftspädagogik auf diese Tätigkeit vorbereitet wurden. Im Rahmen des Schulbandes geht es darum, dass die Studierenden das System Schule erkunden und 


\section{Praxisphasen für das Lehramt an beruflichen Schulen}

unter Begleitung und Anleitung regelmäßig und im Verlauf des Kernpraktikums mit zunehmender Verantwortung eigene Unterrichtseinheiten planen, durchführen und evaluieren.

Tabelle 1 gibt einen Überblick der Berufsbildenden Schulen in Hamburg und Umgebung, die für das Berufsfeld Ernährung und Hauswirtschaft ausbilden oder in der Ausbildungsvorbereitung tätig sind und die sich in den bisher vier Durchgängen des Kernpraktikums beteiligt haben.

Tab. 1: Überblick über die bisher am Kernpraktikum beteiligten Berufsbildenden Schulen in der beruflichen Fachrichtung Ernährungs- und Haushaltswissenschaften (EuH)

\begin{tabular}{|c|c|}
\hline Berufsbildende Schule & Bildungsgänge im Bereich EuH \\
\hline $\begin{array}{l}\text { Berufliche Schule } \\
\text { Elmshorn }\end{array}$ & $\begin{array}{l}\text { Berufsschule: Bäcker/in, Bäckerwerker/in, Fachverkäufer/in } \\
\text { im Nahrungsmittelhandwerk (Schwerpunkte: Fleischerei, } \\
\text { Bäckerei), Fleischer/in, Fachkraft im Gastgewerbe, Fach- } \\
\text { frau/Fachmann für Systemgastronomie, Hotelfachfrau/- } \\
\text { mann, Köchin/Koch, Restaurantfachfrau/-mann } \\
\text { Berufsfachschule: Gesundheit und Ernährung } \\
\text { Berufsgrundbildungsjahr: Gastronomie und Hotellerie } \\
\text { Berufliches Gymnasium Ernährung }\end{array}$ \\
\hline $\begin{array}{l}\text { Berufliche Schule } \\
\text { Recycling- und } \\
\text { Umwelttechnik (G8) }\end{array}$ & Ausbildungsvorbereitung (AV) \\
\hline $\begin{array}{l}\text { Berufliche Schule } \\
\text { Uferstraße (W2) }\end{array}$ & $\begin{array}{l}\text { Berufsschule: Hauswirtschafterin, Fachpraktiker/in } \\
\text { Hauswirtschaft } \\
\text { Berufsfachschule vollqualifizierend: Hauswirtschaft, Haus- } \\
\text { und Familienpflege, Fachpraktiker/in Hauswirtschaft } \\
\text { Berufsvorbereitungsschule }\end{array}$ \\
\hline $\begin{array}{l}\text { Berufsbildende Schule III } \\
\text { Stade }\end{array}$ & $\begin{array}{l}\text { Berufsschule: Hauswirtschafter/in, Fachpraktiker/in Haus- } \\
\text { wirtschaft } \\
\text { Berufsfachschule: Ernährung, Hauswirtschaft und Pflege, } \\
\text { Schwerpunkt: Hauswirtschaft } \\
\text { Fachoberschule: Ernährung und Hauswirtschaft } \\
\text { Fachschule: Hauwirtschaft } \\
\text { Berufseinstiegsklasse: Hauswirtschaft und Pflege } \\
\text { Berufsvorbereitungsjahr:Hauwirtschaft/Agrarwirtschaft } \\
\text { Hauswirtschaft/Gesundheit-Pflege }\end{array}$ \\
\hline $\begin{array}{l}\text { Gewerbeschule Nahrung } \\
\text { und Gastronomie in } \\
\text { Lübeck }\end{array}$ & $\begin{array}{l}\text { Berufsschule: Koch/Köchin, Hotelfachfrau/-mann, } \\
\text { Restaurantfachfrau/-mann, Fachfrau/Fachmann für } \\
\text { Systemgastronomie, Hotelkauffrau-/kaufmann, Fachkraft } \\
\text { im Gastgewerbe, Bäcker/in, Fachverkäufer/in im } \\
\text { Lebensmittelhandwerk (Schwerpunkt: Bäckerei, }\end{array}$ \\
\hline
\end{tabular}




\section{Praxisphasen für das Lehramt an beruflichen Schulen |}

\begin{tabular}{|c|c|}
\hline & $\begin{array}{l}\text { Konditorei, Fleischerei), Konditor/in, Fleischer/in, } \\
\text { Speiseeishersteller/in } \\
\text { Berufsfachschule: Ernährung, Schwerpunkt: Nahrung und } \\
\text { Gaststätten } \\
\text { Fachoberschule: Ernährung/Hauswirtschaft } \\
\text { Berufsoberschule: Ernährung/Hauswirtschaft } \\
\text { Hotelfachschule }\end{array}$ \\
\hline $\begin{array}{l}\text { Staatliche Berufsschule } \\
\text { Eidelstedt (G12) }\end{array}$ & $\begin{array}{l}\text { Fachkraft im Gastgewerbe } \\
\text { Fachpraktikerin Hauswirtschaft }\end{array}$ \\
\hline $\begin{array}{l}\text { Staatliche Gewerbeschule } \\
\text { Ernährung und } \\
\text { Hauswirtschaft (G3) }\end{array}$ & $\begin{array}{l}\text { Berufsschule: Bäckerin, Konditorin, Fachverkäuferin im } \\
\text { Lebensmittelhandwerk (Schwerpunkt Bäckerei/Konditorei) } \\
\text { Berufsfachschule: Gastronomie und Ernährung } \\
\text { Fachoberschule: Ernährung und Hauswirtschaft } \\
\text { Fachschule: Hauswirtschaftliche Dienstleistung } \\
\text { Ausbildungsvorbereitung }(A V)\end{array}$ \\
\hline $\begin{array}{l}\text { Staatliche Gewerbeschule } \\
\text { Gastronomie und } \\
\text { Ernährung (G11) }\end{array}$ & $\begin{array}{l}\text { Berufsschule: Fachkräfte im Gastgewerbe, } \\
\text { Restaurantfachleute, Hotelfachleute, Fachleute in der } \\
\text { Systemgastronomie, Köche und Köchinnen, } \\
\text { Fleischer/innen, Fachverkäufer/innen in der Fleischerei } \\
\text { Berufsfachschule berufsqualifizierend } \\
\text { für gastgewerbliche Ausbildungsberufe } \\
\text { Ausbildungsvorbereitung (AV) }\end{array}$ \\
\hline
\end{tabular}

\section{Unterrichtsversuchsband}

Eingebettet in das Schulband finden im zweiten Semester des Kernpraktikums kollegial hospitierte Unterrichtsversuche in studentischen Kleingruppen statt (Unterrichtsversuchsband), die von Hochschullehrenden des Instituts für Berufs- und Wirtschaftspädagogik (hauptverantwortliche Betreuung) gemeinsam mit Seminarleitungen des Landesinstituts für Lehrerbildung und Schulentwicklung begleitet werden. Dieses Seminar ist nach Fachrichtungen organisiert.

Ein Studierenden-Tandem plant und unterrichtet jeweils eine Doppelstunde in seinem Berufsfach. Dabei orientiert sich das Tandem am schulinternen Curriculum. Freiräume erhält das Tandem bei der inhaltlichen und methodischen Gestaltung der Stunde. Die Studierenden und die Seminarleitung der Fachrichtung hospitieren den Unterricht nach einem vorher festgelegten Hospitationsplan. Jeweils im Anschluss an den hospitierten Unterricht findet die Nachbesprechung des Unterrichts statt.

Bisherige Erfahrungen im Unterrichtsversuchsband zeigen, dass die erste umfassende Planung ihres Unterrichts und das Schreiben eines Unterrichtsentwurfs von den Studierenden als besonders arbeitsintensiv und schwierig erlebt werden. Die gemeinsame Planung und Durchführung des Unterrichts stellt eine Herausforderung 


\section{Praxisphasen für das Lehramt an beruflichen Schulen}

dar, denn es gilt, einen gemeinsamen Nenner zu finden, einer gemeinsamen Planungsstruktur zu folgen, den Gesamtzusammenhang zum Bildungsplan nicht zu verlieren sowie den thematischen Schwerpunkt inhaltlich und methodisch sinnvoll zu gestalten. Die Evaluation des Unterrichtsversuchsbandes zeigt, dass trotz der empfundenen Belastung die eigene Unterrichtsdurchführung und die Reflexion ihres Unterrichts bei den Studierenden als überaus gewinnbringend eingeschätzt werden. Die Studierenden haben die Möglichkeit, sich ausprobieren. Die anschließende Unterrichtsnachbesprechung bietet die Chance, in einer angstfreien Atmosphäre Stärken und Schwächen der Stunde zu analysieren, Handlungsalternativen zu entwickeln und die Perspektive der Schüler einzunehmen.

Die Studierenden gestalten ihren Unterricht in der Regel methodisch vielfältig und einfallsreich. Dazu gehören u. a. sorgfältig ausgearbeitete Medien wie Arbeitsblätter und Filmclips oder kreativ dargestellte Rollenspiele. Was den Kernpraktikanten jedoch sichtlich schwerfällt, ist die Anbindung ihres Themas im Rahmen einer übergreifenden Lernsituation bzw. Problemstellung. Die Studierenden planen ihren Unterricht vorwiegend fachsystematisch (z. B. „Gelier- und Bindemittel“, „Kakao und kakaohaltige Getränke“ „Geflügel"). Sogenannte „Problemstellungen“" werden oft praxisfern konstruiert, sodass am Ende die Lösung des Problems nicht mehr im Vordergrund steht, sondern die Bearbeitung eines Themas mit einer fachsystematisch-umfassenden Struktur.

Die fehlende Problemorientierung und die Schwierigkeit, in Arbeitsprozessen zu denken, sind fast durchgängig Teil der Nachbesprechung des berufsfachlichen Unterrichts. Aufgabe der Seminarleitungen ist es, durch gezielte Fragestellungen anzuregen, dass diese Theorien durch neue Erfahrungen und wissenschaftliche Theorien neu konstruiert werden, damit die Studierenden im Sinne der Lernfelddidaktik professioneller handeln können.

\section{Reflexionsband}

Der Reflexion von Praxiserfahrungen während der unterschiedlichen Formate schulpraktischer Studien im Bachelor- und Masterstudiengang kommt eine besondere Bedeutung zu. Im Rahmen des Reflexionsbandes sollen einerseits anlassbezogen Erfahrungen aus dem Schulband des Kernpraktikums aufgearbeitet, zum anderen aber auch thematische Schwerpunkte theoriebezogen reflektiert sowie spezifische Aspekte der Praxisbegegnung vorbereitet und angebahnt werden. Um diese Prozesse in angemessener Art und Weise begleiten, anregen und unterstützen zu können, wurde für das Kernpraktikum, das die umfangreichste Praxisphase während des Studiums der zukünftigen Lehrkräfte darstellt, in Zusammenarbeit von Kollegen aus dem Institut für Berufs- und Wirtschaftspädagogik und aus dem Landesinstitut für Lehrerbildung und Schulentwicklung (Abteilung Ausbildung Berufsbildende Schulen) ein Konzept entwickelt, das sich an den Erlebensphasen der Studierenden im Kernpraktikum orientiert und eine Ausgestaltung der einzelnen Seminarsitzungen entlang dieser Phasen vorsieht. 


\section{Praxisphasen für das Lehramt an beruflichen Schulen}

Im Reflexionsband trifft sich eine feste Gruppe von Studierenden in der Regel einer beruflichen Fachrichtung etwa 14-tägig, um gemeinsam die Schulerfahrungen zu reflektieren. Die Gruppe wird von je einer Person des Instituts für Berufs- und Wirtschaftspädagogik und des Landesinstituts für Lehrerbildung und Schulentwicklung im Team betreut. In den Veranstaltungen werden anlassbezogen Erfahrungen aus dem Praktikum aufgearbeitet und aus einer theoriebezogenen Perspektive Aspekte der Praxisbegegnung vorbereitet und angebahnt. Dieses Modul wird am Ende des Kernpraktikums durch eine mündliche Prüfung abgeschlossen, deren Grundlage ein Präsentationsportfolio darstellt.

\section{Zur Makrostruktur des Reflexionsbandes}

Als Orientierungsgrundlage bei der Planung des Reflexionsbandes dienen zwei fiktive Studierende - „Ole“ und „Olga“ -, die geschaffen wurden, um die Perspektive der Studierenden bei der Konzeption des Reflexionsbandes und des Kernpraktikums insgesamt nicht aus dem Blick zu verlieren. Ole und Olga sollen behilflich sein, sich vor allem die Studien- und Lebenssituation zu vergegenwärtigen, um diese bei der Planung der einzelnen Bänder angemessen zu berücksichtigen (Tramm et al., 2012).

Für die Konkretisierung sowohl der inhaltlichen Ausrichtung des Reflexionsbandes als auch der Arbeit in den einzelnen Seminarsitzungen wurden Ole und Olga herangezogen, um zu antizipieren, welche Erfahrungen der Studierenden an den Schulen zu welchen Zeitpunkten im Kernpraktikum im Vordergrund stehen würden. Vor dem Hintergrund dieser Überlegungen wurden fünf studentische Erlebensphasen während des Kernpraktikums identifiziert:

1. Orientierung im Kernpraktikum

2. Kennenlernen der Schule und Planung des Praktikums

3. Hospitationen und Assistenztätigkeiten

4. eigener Unterricht

5. Abschluss des Kernpraktikums

Diese fünf Phasen sollen das Erleben der Studierenden im Schulband widerspiegeln. Anhand der Phasen werden Schwerpunkte deutlich, von denen angenommen wird, dass diese für die Kernpraktikanten in der jeweiligen Phase an der Schule im Vordergrund stehen.

Die Erlebensphasen dienen als Grundlage für die Planung des Reflexionsbandes. Für jede der insgesamt zwölf Seminarsitzungen wurden folgende Aspekte ausgearbeitet:

- Schwerpunkt (z. B. Orientierung im Kernpraktikum und an der Schule),

- Termin der Seminarsitzung,

- Fokus der Seminarsitzung (z. B. Lehrer/-innen und Lehrhandeln, Lehrer/ -innenrolle),

- antizipierte Beobachtungen und Erfahrungen der Studierenden an der Schule (z. B. Unterrichtserfolge oder -misserfolge), 


\section{Praxisphasen für das Lehramt an beruflichen Schulen}

- erwartetet Themen für die erfahrungsbezogene Reflexionsphase im Seminar (z. B. Unterschiedlichkeit von Klassen, Lehrkräften und erlebten Unterrichten, kommunikative Erfahrungen mit Schüler/-innen, Berichte über eigenen Unterricht und Probleme bei der Vorbereitung),

- Ziel der Seminararbeit (z. B. eigene Stärken, Schwächen und Entwicklungsbedarfe benennen können),

- Themenangebot im Seminar (z. B. Individualisierungs- und Differenzierungsmöglichkeiten),

- Handlungs- und Beobachtungsaufträge für die folgenden Schultermine (z. B. Erstellen eines Schülerportraits).

Über die Planungsaspekte für die jeweiligen Seminarsitzungen ergeben sich zwei zentrale Reflexionsphasen für jede Sitzung und damit die Mikrostrukturierung des Seminars (vgl. Abbildung 3).

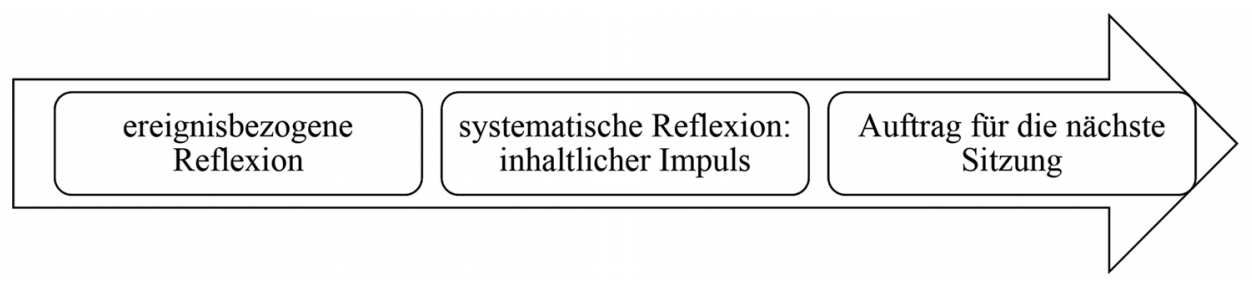

Abb. 3: Phasierung der Seminarsitzungen im Reflexionsband des Kernpraktikums Lehramt an beruflichen Schulen

\section{Zur Mikrostruktur der Seminarsitzungen im Reflexionsband}

$\mathrm{Zu}$ Beginn der Seminarsitzung findet eine erfahrungsbezogene Reflexion statt, in der die Studierenden Themen einbringen, die für sie persönlich aufgrund ihrer Schulerfahrungen eine besondere Relevanz besitzen. Im Zuge der Planung des Reflexionsbandes wurden Überlegungen dazu angestellt, welche Erfahrungen „Ole“ und „Olga“ während der unterschiedlichen Erlebensphasen an der Schule sammeln, und welche Themen demzufolge in dieser Reflexionsphase von den Studierenden eingebracht werden könnten (Aspekte in der Übersicht: antizipierte Beobachtungen und Erfahrungen der Studierenden an der Schule sowie erwartete Themen für die erfahrungsbezogene Reflexionsphase im Seminar).

Im Anschluss an diese Reflexionsphase findet eine systematische Reflexion mit Bezug auf einen vorgegebenen thematischen Schwerpunkt statt, wobei sich in der Seminararbeit an einem Verfahren in fünf Schritten nach Korthagen (2002, S. 160 ff.) orientiert wird:

1. Vorstrukturierung:

Die Studierenden erhalten am Ende einer jeden Seminarsitzung einen Handlungs- und Beobachtungsauftrag, den sie bis zur nächsten Seminarsitzung bearbeiten sollen. Mit diesem Auftrag wird darauf abgezielt, dass die Studierenden spezifische Erfahrungen an der Schule sammeln, z. B. Anfertigen eines Unterrichtsnarrativs. 


\section{Praxisphasen für das Lehramt an beruflichen Schulen}

2. Erfahrung:

Die Studierenden gehen an die Schule und bearbeiten den Handlungsund Beobachtungsauftrag, sammeln also vorstrukturierte Erfahrungen.

3. Strukturierung:

Die Studierenden bringen ihre Erfahrungen im Reflexionsseminar ein und tauschen sich gegenseitig aus. Im Zuge dessen erfolgt eine Strukturierung der Erfahrungen der Studierenden.

4. Fokussierung:

Im vorangegangenen Schritt haben sich idealtypisch unterschiedliche Aspekte herausgebildet, die vertieft werden können. In diesem Schritt erfolgt eine Fokussierung auf einen bestimmten Schwerpunkt.

5. Theorie:

In diesem fünften Verfahrensschritt erfolgt der Einbezug theoretischer Konzepte und Modelle, in dem sich theoriegeleitet mit dem im Schritt vier identifizierten Schwerpunkt auseinandergesetzt wird.

Unter Berücksichtigung der Erlebensphasen wurden für die jeweiligen Reflexionssitzungen thematische Schwerpunkte festgelegt, die in allen Seminaren behandelt werden.

Es wird deutlich, dass es sich überwiegend um pädagogische und nicht zwangsläufig um fachdidaktische Themen handelt. Diese werden überwiegend im Rahmen des Unterrichtsversuchsbandes thematisiert und dort, wo es sich anbietet, werden die Themen im Reflexionsband, auch bedingt durch die Kooperation der Seminarleitung aus dem Institut für Berufs- und Wirtschaftspädagogik und dem Landesinstitut für Lehrerbildung und Schulentwicklung, in einen fachrichtungsspezifischen Kontext gebracht.

Tab. 2: Überblick über die thematischen Schwerpunkte in den Reflexionssitzungen

\begin{tabular}{l|l|l}
\hline Sitzung & Erlebensphase & Thematischer Schwerpunkt \\
\hline 1 & Orientierung im Kernpraktikum & Orientierung im Kernpraktikum \\
\hline 2 & $\begin{array}{l}\text { Kennenlernen der Schule und Prak- } \\
\text { tikumsplanung }\end{array}$ & $\begin{array}{l}\text { Orientierung der Schule und } \\
\text { Praktikumsplanung }\end{array}$ \\
\hline 3 & Hospitationen, Assistenztätigkeiten & Unterricht ganzheitlich erleben \\
\hline 4 & Hospitationen, Assistenztätigkeiten & $\begin{array}{l}\text { Ansprüche an Unterricht (Mentoren } \\
\text { und selbst) }\end{array}$ \\
\hline 5 & Hospitationen, Assistenztätigkeiten & $\begin{array}{l}\text { Hospitationsstrategien - Unterricht } \\
\text { verstehen }\end{array}$ \\
\hline 7 & Hospitationen, Assistenztätigkeiten & Schülerportraits - Heterogenität \\
\hline 8 & eigener Unterricht & Didaktisches Selbstverständnis \\
\hline & eigener Unterricht & $\begin{array}{l}\text { Unterrichtsstörungen - } \\
\text { Klassenführung }\end{array}$ \\
\hline
\end{tabular}




\section{Praxisphasen für das Lehramt an beruflichen Schulen}

\begin{tabular}{l|l|l}
\hline 9 & eigener Unterricht & Individualisierung und Inklusion \\
\hline 10 & eigener Unterricht & Innovationen an Schulen \\
\hline 11 & eigener Unterricht & $\begin{array}{l}\text { Reflexion von Problemen im eigenen } \\
\text { Unterricht }\end{array}$ \\
\hline 12 & Abschluss Kernpraktikum & $\begin{array}{l}\text { Kernpraktikum als Teil des } \\
\text { Professionalisierungsprozesses }\end{array}$ \\
\hline
\end{tabular}

\section{Seminarband}

Im Seminarband werden den Studierenden über die zwei Semester des Kernpraktikums hinweg „Qualifizierungsbausteine“ angeboten, durch die sie in der Bewältigung der praktischen Anforderungen des Schulbandes unterstützt werden sollen sowie Angebote in der Fachdidaktik ihres Unterrichtsfaches. Zudem soll ihnen die Möglichkeit gegeben werden, an wahrgenommenen Entwicklungsbedarfen zu arbeiten oder spezifische Fragen und Interessen zu vertiefen. Die Anbieter solcher Qualifizierungsbausteine sind Fachdidaktiker/-innen der Universität und (Fach-) Seminarleiter/-innen des Landesinstituts für Lehrerbildung und Schulentwicklung (Abteilung Ausbildung Berufsbildende Schulen).

\section{Projektband}

Mit dem Projektband verbindet sich die Idee, forschendes Lernen im Kernpraktikum anzubahnen. Im Rahmen des Kernpraktikums sollen die Studierenden ein Forschungsprojekt durchführen, sie sollen sich in diesem mit gehaltvollen Problemen pädagogischer Praxis theoriegeleitet auseinandersetzen und Forschungsfragen systematisch bearbeiten. Das Projektband wird von Lehrenden des Instituts für Lehrerbildung und Schulentwicklung ggf. in Kooperation mit den Fachwissenschaften betreut. Die Studierenden schließen dieses Modul am Ende des Kernpraktikums mit einer Präsentation ihres Projekts sowie einer schriftlichen Ausarbeitung ab. Das Projektband ist fachrichtungsübergreifend organisiert. Im Folgenden sind exemplarisch die Projekte der Studierenden der beruflichen Fachrichtung Ernährungs- und Haushaltswissenschaften für den Durchgang im Sommersemester 2014 und im Wintersemester 2014/2015 aufgeführt:

- Schülerrückmeldung als Evaluationsansatz - Qualitative Interviews zum Pilotprojekt: „Berufsgrundbildungsjahr Gastronomie und Hotellerie““

- Einführung in das Selbstorganisierte Lernen

- Eigenschaften und Merkmale von Schülern und Schülerinnen der Ausbildungsvorbereitung, die für Betriebe der Handelsbranche für positive Praktika und Übergänge in die Berufsausbildung bedeutend sind

- Einstellungen zur erfolgreichen Umsetzung des Inklusionsgedankens an einer Hamburger Berufsschule 


\section{Praxisphasen für das Lehramt an beruflichen Schulen}

- Entwicklung einer Handreichung und Durchführung eines Workshop in der Lehrerfortbildung zum Thema Leichte Sprache in der Unterrichtskommunikation

- Entwicklung didaktischer Materialien für einen Förderschulkurs „Kochen" unter besonderer Berücksichtigung der Leichten Sprache

- Inklusion - Schulung für zukünftige Lehrer zur Information über und Sensibilisierung für ausgewählte Behinderungen vor dem Hintergrund eines inklusiven Unterrichts

- Das Lehrerzimmer als Kommunikationstreffpunkt

- Interkulturelle Bildung und Erziehung - eine Analyse der Rahmenlehrpläne gastronomischer Berufe zur Ermittlung möglicher Anknüpfungspunkte im Unterricht.

\section{Fazit}

Mittlerweile befindet sich der vierte Durchgang von Studierenden im Kernpraktikum. ${ }^{1}$ Die Erfahrungen zeigen, dass sich das Konzept des Kernpraktikums bewährt, wenngleich sehr deutlich wird, dass dieses für die Studierenden mit einer hohen Komplexität verbunden ist, und dessen Bewältigung auf unterschiedlichen Ebenen teilweise eine besondere Herausforderung darstellt.

Im Hinblick auf das Reflexionsband im Kernpraktikum hat sich sowohl aus der Perspektive der Lehrenden als auch aus der Perspektive der Studierenden die Orientierung an den Erlebensphasen bewährt. Zudem wird die Phasierung der einzelnen Seminarsitzungen grundsätzlich positiv eingeschätzt, allerdings besteht durchaus die Gefahr, dass der erfahrungsbezogenen Reflexion zu Beginn der Seminarsitzungen zu wenig Zeit eingeräumt wird.

Eine besondere Herausforderung im Kontext des Reflexionsbandes kann zum einen darin gesehen werden, individuell auf die Entwicklungsbedarfe der Studierenden einzugehen. Zum anderen wird deutlich, dass es für die Anbahnung einer theoriegeleiteten Reflexion entscheidend ist, welche theoretischen Bezugspunkte herangezogen werden und inwiefern sich hierfür vor dem Hintergrund der studentischen Erfahrungen Anknüpfungspunkte ergeben.

\section{Anmerkung}

1 Die Einführung des Kernpraktikums wird im Rahmen einer formativen Evaluation systematisch begleitet. In der Evaluation wird u. a. der Fragestellung nachgegangen, wie Studierende dieses neue Praxiselement subjektiv erleben und welche Strategien sie anwenden, um dieses erfolgreich zu gestalten und es mit ihrem weiteren Studium und mit ihren individuellen Lebenskontexten zu vereinbaren. Zudem werden die Wahrnehmungen und Beurteilungen dieser Praxisphase aus der Perspektive der anderen Beteiligten evaluiert, der Lehrenden aus dem Institut für Berufs- und Wirtschaftspädagogik und dem Landesinstitut für Lehrerbildung 


\section{Praxisphasen für das Lehramt an beruflichen Schulen}

und Schulentwicklung (Abteilung Ausbildung Berufsbildende Schulen) sowie der Schulen.

\section{Literatur}

Behörde für Schule und Berufsbildung/HIBB (Hrsg.) (2014). Berufswunsch: Lehrerin oder Lehrer an einer berufsbildenden Schule in Hamburg. Hamburg.

Korthagen, F. A. J. (2002). Schulwirklichkeit und Lehrerbildung. Reflexion der Lehrertätigkeit. Hamburg: ebv.

Naeve-Stoß, N. (2013). Studienreform aus studentischer Perspektive - Einzelfallstudien zur Rekonstruktion studentischer Wahrnehmungen, Beurteilungen und Studienstrategien im Rahmen des Lehramtsstudiums für berufliche Schulen. Paderborn: Eusl.

Riebenbauer, E./Naeve-Stoß, N. (2013). Studierst du noch oder reflektierst du schon? Anspruch und Realität im Schulpraktikum. Wissenplus, 5, 31-36.

Tramm, T. \& Naeve, N. (2010). Ganzheitlich curriculares und didaktisches Konzept für die Berufliche Fachrichtung ,Wirtschaft und Verwaltung“ - Kompetenzdimensionen, Entwicklungsverlauf, Entwicklungsaufgaben. In J.-P. Pahl \& V. Herkner (Hrsg.), Handbuch Berufliche Fachrichtungen (S. 291-310). Bielefeld: wbv.

Tramm, T., Fahland, B. \& Naeve, N. (2012). Das Hamburger Kernpraktikum - ein innovativer Ansatz zur Verknüpfung von Praxis- und Forschungsorientierung in der Lehrerbildung. In Bundesarbeitskreis der Seminar- und Fachleiter/innen e. V. (BAK) (Hrsg.), Seminar - Lehrerbildung und Schule 1/2012. Wege zur Professionalisierung - 45. Seminartag Jena (S. 105-117). Jena: Schneider .

\section{Verfasserinnen}

Dr. ${ }^{\text {in }}$ Nicole Naeve-Stoß

Dr. ${ }^{\text {in }}$ Julia Kastrup

Universität Hamburg

Institut für Berufs- und

Wirtschaftspädagogik

Sedanstraße 19,

D-20146 Hamburg

E Mail: nicole.naeve@uni-hamburg.de; julia.kastrup@uni-hamburg.de
Gabriele Herold

Landesinstitut für Lehrerbildung und

Schulentwicklung,

Abteilung 3,

Berufsbildende Schulen

Felix-Dahn-Straße 3

D-20357 Hamburg

E-Mail: gabriele.herold@li-hamburg.de 